\title{
Oncological Risk of Colonic Stents Used as Bridge to Surgery for Left-Side Malignant Colonic Obstructions
}

\author{
Takeshi Yamada, MD, PhD, Akihisa Matsuda, MD, PhD, Goro Takahashi, MD, PhD, and \\ Hiroshi Yoshida, MD, PhD
}

Department of Gastrointestinal and Hepato-Biliary-Pancreatic Surgery, Nippon Medical School, Tokyo, Japan

\begin{abstract}
Management of acute left-side malignant colonic obstruction (LMCO) remains controversial. Emergency colorectal surgery for LMCO is associated with high mortality. Self-expanding metallic stents (SEMS) are used as an alternative to emergency LMCO surgery. In 2007, a systemic review reported that patients with a bridge to surgery (BTS) had lower morbidity and mortality rates in subsequent operations. ${ }^{1}$ However, in 2011, a randomized trial found that BTS offered no definitive clinical advantage over emergency surgery. ${ }^{2}$ In 2013, a retrospective study conducted by Sabbagh et al. warned that overall survival of patients with BTS can be shorter than that of patients who receive immediate surgery. ${ }^{3}$ In addition, a metaanalysis showed that BTSs significantly increase the risk of overall recurrence (relative risk 1.4) and systemic recurrence (relative risk 1.6). ${ }^{4}$ Conversely, a metaanalysis found that overall survival of BTS patients is equal to that of patients receiving emergency surgery. ${ }^{5}$
\end{abstract}

How BTSs might worsen survival of patients with LMCO remains unclear. We previously reported that SEMS placement increases plasma levels of circulating cell-free DNA (cfDNA) and circulating tumor DNA (ctDNA) resulting from tumor manipulation. ${ }^{6}$ Although a randomized control study (JCOG 1006) failed to show a clear disadvantage, tumor manipulation may worsen prognoses of patients with malignant disease. In that study, 7 days after insertion of SEMS, cfDNA concentration was

(C) Society of Surgical Oncology 2022

First Received: 24 May 2021

Accepted: 14 December 2021;

Published Online: 4 January 2022

T. Yamada, MD, PhD

e-mail: y-tak@nms.ac.jp significantly higher than before insertion, and cfDNA concentration 7 days after insertion showed a strong positive correlation with ctDNA and serum lactate dehydrogenase levels. This indicates that SEMS injure cancer cells and disperse damage-associated molecular patterns (DAMPs), which potently trigger sterile inflammation. DAMPs represent a large range of chemically unrelated mediators such as High Mobility Group Box 1 (HMGB1), S100 proteins, hyaluronan, and heat-shock proteins. DAMPs are released from necrotic cells. Uniform DNA fragments of 185-200 bp are released from apoptotic cells. In contrast, DNA fragments of various lengths are released from necrotic cells because of random and incomplete digestion of genomic DNA by deoxyribonucleases. ${ }^{7}$ Thus, necrotic cells are the source of longfragment cfDNA (lcfDNA). In our previous study, ${ }^{6}$ large amounts of lcfDNA were detected after SEMS insertion, indicating that SEMS cause necrotic cell death, resulting in sterile inflammation induced by DAMPs.

In this issue of Annals of Surgical Oncology, Broholm et al. clearly show that SEMS placement induces unfavorable gene expression changes in the tumor microenvironment associated with tumor progression in LMCO patients. ${ }^{8}$ These unfavorable genes are associated with tumor proliferation, migration, invasion, angiogenesis, and inflammation. The contents of necrotic cells enhance angiogenesis and proliferation of endothelial cells. ${ }^{9}$ Therefore, this study moves us one step closer to understanding how BTSs can worsen survival of LMCO patients. In addition, mechanical compression enhances angiogenesis via miR-9 downregulation, which upregulates production of vascular endothelial growth factors. ${ }^{10}$ That is, SEMS can induce necrotic cell death, which is 
associated with inflammation, angiogenesis, and cell proliferation. Angiogenesis is also induced by mechanical compression of SEMS.

Despite inducing unfavorable gene expression changes, BTSs certainly decrease mortality and morbidity. The interval between SEMS placement and surgery can also impact prognosis. Based on these principles, further discussion of benefits and detriments of BTS is needed. Patients should be informed about the potential oncological risk posed by SEMS before physicians employ them.

DISCLOSURE The authors declare no conflicts of interest.

\section{REFERENCES}

1. Breitenstein S, Rickenbacher A, Berdajs D, Puhan M, Clavien PA, Demartines N. Systematic evaluation of surgical strategies for acute malignant left-sided colonic obstruction. $\mathrm{Br} J$ Surg. 2007;94(12):1451-60.

2. Van Hooft JE, Bemelman WA, Oldenburg B, et al. Colonic stenting versus emergency surgery for acute left-sided malignant colonic obstruction: a multicentre randomised trial. Lancet Oncol. 2011;12(4):344-52.

3. Sabbagh C, Browet F, Diouf M, et al. Is stenting as "a bridge to surgery" an oncologically safe strategy for the management of acute, left-sided, malignant, colonic obstruction? A comparative study with a propensity score analysis. Ann Surg. 2013;258(1):107-15.

4. Foo CC, Poon SHT, Chiu RHY, Lam WY, Cheung LC, Law WL. Is bridge to surgery stenting a safe alternative to emergency surgery in malignant colonic obstruction: a meta-analysis of randomized control trials. Surg Endosc. 2019;33(1):293-302.

5. Matsuda A, Miyashita M, Matsumoto S, et al. Comparison of long-term outcomes of colonic stent as "bridge to surgery" and emergency surgery for malignant large-bowel obstruction: a meta-analysis. Ann Surg Oncol. 2015;22(2):497-504.

6. Takahashi G, Yamada T, Iwai T, et al. Oncological assessment of stent placement for obstructive colorectal cancer from circulating cell-free DNA and circulating tumor DNA dynamics. Ann Surg Oncol. 2018;25(3):737-44.

7. Wang BG, Huang HY, Chen YC, et al. Increased plasma DNA integrity in cancer patients. Cancer Res. 2003;63(14):3966-8.

8. Kobborg M, Broholm M, Frostberg E, Jeppesen M, Gögenür I. Short-term results of self-expanding metal stents for acute malignant large bowel obstruction. Colorectal Dis. 2017;19(10):O365-o371.

9. Karsch-Bluman A, Feiglin A, Arbib E, et al. Tissue necrosis and its role in cancer progression. Oncogene. 2019;38(11):1920-35.

10. Kim BG, Gao MQ, Kang S, et al. Mechanical compression induces VEGFA overexpression in breast cancer via DNMT3Adependent miR-9 downregulation. Cell Death Dis. 2017;8(3):e2646.

Publisher's Note Springer Nature remains neutral with regard to jurisdictional claims in published maps and institutional affiliations. 